CRYSTALLOGRAPHIC COMMUNICATIONS

ISSN 2056-9890

Received 11 June 2016

Accepted 7 July 2016

Edited by S. Parkin, University of Kentucky, USA

Keywords: crystal structure; substituted 2-azabuta-1,3-diene; weak hydrogen bonding;

halogen bonding; $\pi-\pi$ interactions .

CCDC reference: 1491488

Supporting information: this article has supporting information at journals.iucr.org/e

\section{Crystal structure of 4,4-dibromo-1-(3,4-dimethoxy- phenyl)-2-azabuta-1,3-diene-1-carbonitrile}

\author{
Marwa Chaabene, ${ }^{a}$ Abderrahim Khatyr, ${ }^{b}$ Michael Knorr, ${ }^{b} *$ Moheddine Askri, ${ }^{a}$ \\ Yoann Rousselin ${ }^{\mathrm{c}}$ and Marek M. Kubicki ${ }^{\mathrm{c} *}$
}

\begin{abstract}
a'Laboratoire de Chimie Hétérocyclique, Produits Naturels et Réactivité (LR11ES39), Département de Chimie, Faculté des Sciences de Monastir, Tunisia, ${ }^{\mathbf{b}}$ Institut UTINAM UMR CNRS 6213, University of Franche-Comté, 16 route de Gray, 25030 Besançon, France, and ' ICMUB UMR CNRS 6302, University of Bourgogne, 9 avenue Alain Savary, 21078 Dijon, France.*Correspondence e-mail: michael.knorr@univ-fcomte.fr, marek.kubicki@u-bourgogne.fr
\end{abstract}

The title compound, $\mathrm{C}_{12} \mathrm{H}_{10} \mathrm{Br}_{2} \mathrm{~N}_{2} \mathrm{O}_{2}$, represents an example of a planar $\pi$ conjugated 2-azabutadiene molecule, which is both an interesting starting material for further organic transformations and a potential ligand in organometallic coordination chemistry. Its metric molecular parameters are typical for the family of 2-azabuta-1,3-dienes not substituted at the $(\mathrm{CH}) 3$-position. In the crystal, the almost planar (r.m.s. deviation $=0.0658 \AA$ ) azadiene molecules form onedimensional double-wide ribbons through intermolecular halogen bonds ( $\mathrm{C}-$ $\mathrm{Br} \cdots \mathrm{O}$ and $\mathrm{C}-\mathrm{Br} \cdots \mathrm{Br}-\mathrm{C}$ ), which then stack in a slipped manner through weak $\mathrm{C}-\mathrm{H} \cdots \mathrm{Br}$ and $\pi-\pi$ interactions to generate a three-dimensional network.

\section{Chemical context}

In the context of our interest in developing novel $\pi$-conjugated dithioether compounds as ligands for coordination chemistry and further organic transformations, we have reported on the synthesis and crystal structure of 4,4-dichloro1,1-diphenyl-2-azabuta-1,3-diene $\quad\left[\mathrm{Ph}_{2} \mathrm{C}=\mathrm{N}-\mathrm{C}(\mathrm{H})=\mathrm{CCl}_{2}\right]$ and its conversion to $\left[\mathrm{Ph}_{2} \mathrm{C}=\mathrm{N}-\mathrm{C}(\mathrm{H})=\mathrm{C}(\mathrm{SR})_{2}\right]$ and $\left[\mathrm{Ph}_{2} \mathrm{C}=\mathrm{N}-\mathrm{C}(\mathrm{H})=\mathrm{C}(\mathrm{OPh})_{2}\right]$ by reaction with thiolates $\mathrm{NaS} R$ or $\mathrm{NaOPh}$, respectively (Jacquot et al., 1999, 2000; JacquotRousseau et al., 2006; Kinghat et al., 2016). Several crystal structures of these molecules/ligands and their derived transition metal complexes reveal that despite the overall planarity of the $\pi$-conjugated chain, one aryl group of the $-\mathrm{N}=\mathrm{CPh}_{2}$ imine segment is tilted with respect to the azabutadienic array (Jacquot et al., 1999; Knorr et al., 2003; Kinghat et al., 2008). To circumvent this feature and to modulate the stereoelectronic properties, we examined other synthetic strategies for the synthesis of 2-azabutadienes. Intrigued by a communication briefly mentioning the formation of the nitrile-functionalized compounds $[\mathrm{Ph}(\mathrm{C} \equiv \mathrm{N}) \mathrm{C}=$ $\left.\mathrm{N}-\mathrm{C}(\mathrm{H})=\mathrm{C} X_{2}\right](X=\mathrm{Cl}$ or $\mathrm{Br})$ by treatment of the

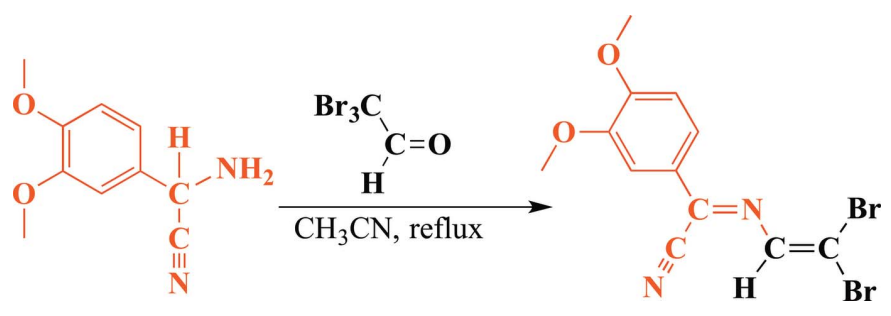

Figure 1

The reaction scheme for the synthesis of (1). 


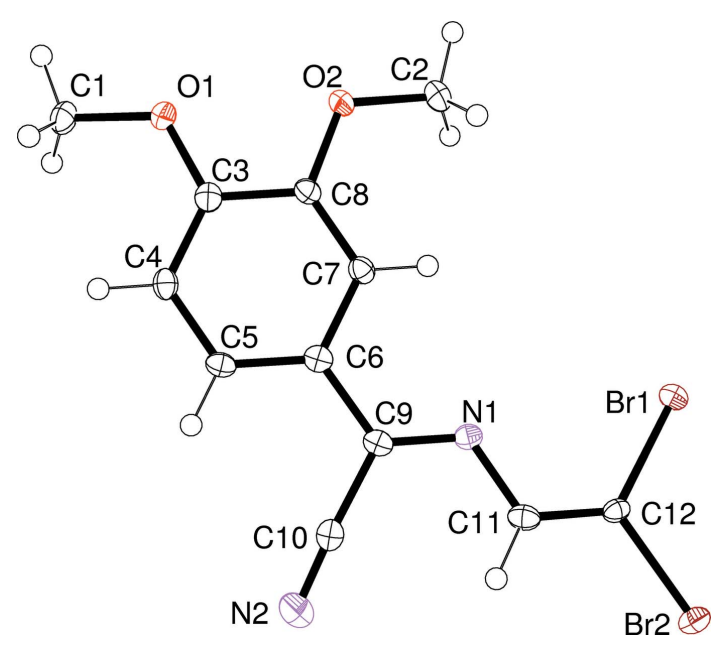

Figure 2

An displacement ellipsoid plot of (1) at the 50\% probability level.

$\alpha$-aminonitrile $\mathrm{H}_{2} \mathrm{NCHPhC} \equiv \mathrm{N}$ with chloral or bromal (Sato $\&$ Adachi, 1978), we reinvestigated this reaction to explore the scope for the synthesis of other derivatives. For example, we succeeded in preparing the title compound $\left[\mathrm{C}_{6} \mathrm{H}_{3}(\mathrm{OMe})_{2}\right.$ $\left.(\mathrm{C} \equiv \mathrm{N}) \mathrm{C}=\mathrm{N}-\mathrm{C}(\mathrm{H})=\mathrm{CBr}_{2}\right]$, (1), bearing two electrondonating methoxy groups at the meta- and para-positions of the aryl ring (see Fig. 1).<smiles>COc1ccc(/C(C#N)=N/C=C(Br)Br)cc1OC</smiles>

\section{Structural commentary}

Compound (1) crystallizes from acetonitrile in the triclinic crystal system, space group $P \overline{1}$. The transoid conformation of the azabutadiene chain found in $\left[\mathrm{Ph}_{2} \mathrm{C}=\mathrm{N}-\mathrm{C}(\mathrm{H})=\mathrm{CCl}_{2}\right]$ (Jacquot et al., 1999) is also observed in the crystal structure of (1) (Fig. 2). The azadiene chain (C9/N1/C11/C12) is essentially
Table 1

Halogen-bonding parameters $\left(\AA,^{\circ}\right)$ for $(1)$.

\begin{tabular}{llllll}
\hline$D$ & $\mathrm{Br}$ & $A$ & $D-\mathrm{Br}$ & $\mathrm{Br} \cdots A$ & $\mathrm{D}-\mathrm{Br} \cdots \mathrm{A}$ \\
\hline $\mathrm{C} 12$ & $\mathrm{Br} 2$ & $\mathrm{O} 1^{\mathrm{i}}$ & $1.878(3)$ & $3.185(2)$ & $124.26(9)$ \\
$\mathrm{C} 12$ & $\mathrm{Br} 2$ & $\mathrm{O}^{\mathrm{i}}$ & $1.878(3)$ & $3.153(2)$ & $167.6(1)$ \\
$\mathrm{C} 12$ & $\mathrm{Br} 1$ & $\mathrm{Br}^{\mathrm{ii}}$ & $1.872(3)$ & $3.4340(5)$ & $144.8(1)$ \\
\hline
\end{tabular}

Symmetry codes: (i) $x-1, y, z-1$; (ii) $-x+1,-y+2,-z+1$.

Table 2

Hydrogen-bond geometry $\left(\AA,^{\circ}\right)$.

\begin{tabular}{lllll}
\hline$D-\mathrm{H} \cdots A$ & $D-\mathrm{H}$ & $\mathrm{H} \cdots A$ & $D \cdots A$ & $D-\mathrm{H} \cdots A$ \\
\hline $\mathrm{C} 1-\mathrm{H} 1 A \cdots \mathrm{Br} 1^{\mathrm{i}}$ & 0.98 & 3.01 & $3.867(4)$ & 146 \\
$\mathrm{C} 1-\mathrm{H} 1 B \cdots \mathrm{Br}^{\mathrm{ii}}$ & 0.98 & 3.04 & $3.869(4)$ & 143 \\
\hline
\end{tabular}

Symmetry codes: (i) $-x+1,-y+1,-z+1$; (ii) $-x,-y+1,-z+1$.

planar (r.m.s. deviation $=0.014 \AA$ ). The torsion angle $\mathrm{C} 12-$ $\mathrm{C} 11-\mathrm{N} 1-\mathrm{C} 9$ is $177.9(3)^{\circ}$. The aryl ring, as well as the $\mathrm{CN}$ substituent, form part of the $\pi$-conjugated array. The length of the vinylic $\mathrm{C} 11=\mathrm{C} 12$ bond matches well with that of $\left[\mathrm{Ph}_{2} \mathrm{C}=\mathrm{N}-\mathrm{C}(\mathrm{H})=\mathrm{CCl}_{2}\right][1.332$ (4) versus 1.319 (3) $\AA$ ]. We are not aware of any other structurally characterized azabutadienes bearing a $\mathrm{Br}_{2} \mathrm{C}=\mathrm{C}$ moiety. For other organic compounds containing this dibromovinyl unit, such as 2,2-dibromovinylthiophene and 2-(2,2-dibromovinyl)-1-methyl-1Himidazole-4,5-dicarbonitrile, $\mathrm{C}=\mathrm{C}$ distances of 1.335 (7) and 1.317 (3) § have been reported (Clément et al., 2011; Lokaj et al., 2011). The $\mathrm{C} 9=\mathrm{N} 1$ bond length of the imine group is also comparable with that of $\left[\mathrm{Ph}_{2} \mathrm{C}=\mathrm{N}-\mathrm{C}(\mathrm{H})=\mathrm{CCl}_{2}\right][1.288$ (3) versus 1.293 (2) ̊].

\section{Supramolecular features}

Each planar molecule of (1) is connected through halogen (Cavallo et al., 2016) bifurcated bonds $\mathrm{C} 12-\mathrm{Br} 2 \cdots(\mathrm{O} 1, \mathrm{O} 2)$ to two neighbouring molecules to form a one-dimensional ribbon. The ribbon is further connected through another kind of side halogen bond $(\mathrm{C} 12-\mathrm{Br} 1 \cdots \mathrm{Br} 1-\mathrm{C} 12)$ to other neighbouring molecules with the formation of roughly planar one-dimensional double-wide straight chains (Fig. 3 and Table 1). These chains then stack in a slipped manner through

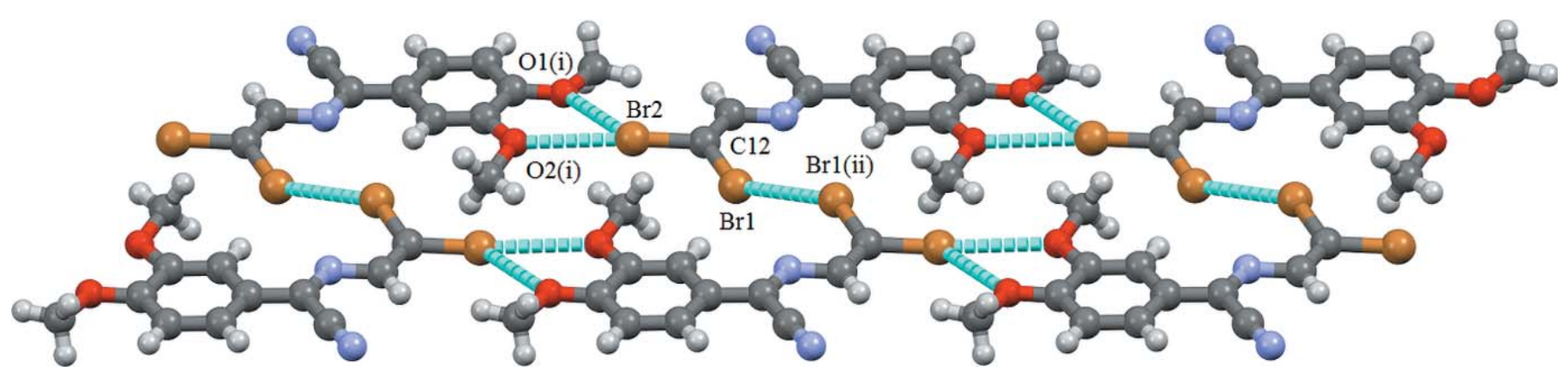

Figure 3

Part of the crystal structure of (1), showing the formation of double-wide ribbons through halogen $\mathrm{C}-\mathrm{Br} \cdots \mathrm{O}$ and $\mathrm{C}-\mathrm{Br} \cdots \mathrm{Br}-\mathrm{C}$ bonding. [Symmetry codes: (i) $x-1, y, z-1$; (ii) $-x+1,-y+2,-z+1$.] 
Table 3

$\pi-\pi$ interactions $(\AA)$ in (1).

\begin{tabular}{llllll}
\hline Atom $A$ & Atom $B$ & $A \cdots B$ & Atom $C$ & Atom $D$ & $C \cdots \mathrm{D}$ \\
\hline $\mathrm{C} 5$ & $\mathrm{C}^{2} 2^{\mathrm{ii}}$ & $3.445(5)$ & $\mathrm{C} 11$ & $\mathrm{O}^{\mathrm{i}}$ & $3.455(4)$ \\
$\mathrm{C} 6$ & $\mathrm{C} 11^{\mathrm{ii}}$ & $3.497(5)$ & $\mathrm{N} 1$ & $\mathrm{C}^{\mathrm{i}}$ & $3.556(4)$ \\
$\mathrm{C} 9$ & $\mathrm{~N}^{\mathrm{ii}}$ & $3.451(4)$ & $\mathrm{C} 9$ & $\mathrm{C} 8^{\mathrm{i}}$ & $3.523(5)$ \\
& & & $\mathrm{C} 6$ & $\mathrm{C}^{\mathrm{i}}$ & $3.559(5)$ \\
\hline
\end{tabular}

Symmetry codes: (i) $1-x, 1-y, 1-z$; (ii) $-x, 1-y, 1-z$.

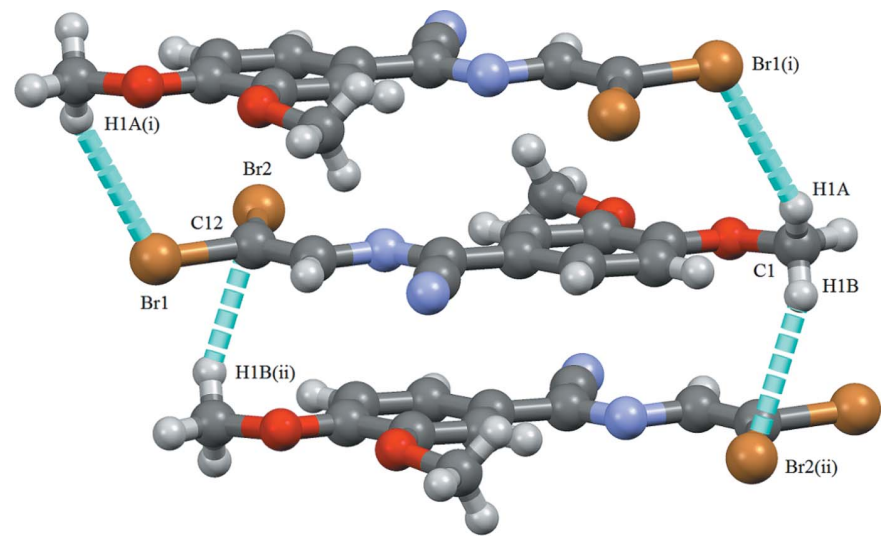

Figure 4

Part of the crystal structure of (1), showing the $\mathrm{C}-\mathrm{H} \cdots \mathrm{Br}$ interactions. [Symmetry codes: (i) $-x+1,-y+1,-z+1$; (ii) $-x,-y+1,-z+1$.]

very weak $\mathrm{C}-\mathrm{H} \cdots \mathrm{Br}$ interactions (Fig. 4 and Table 2) to generate a three-dimensional supramolecular network (Fig. 5). When projecting the structure down the direction perpendicular to the planes of the planar molecules of (1) (e.g. down from the top in Fig. 4), one sees an interesting overlap in a head-to-tail arrangement of zigzagging unsaturated chains that leads to the formation of $\pi-\pi$ stacking interactions around the symmetry centres located at $\left(0, \frac{1}{2}, \frac{1}{2}\right)$ and $\left(\frac{1}{2}, \frac{1}{2}, \frac{1}{2}\right)$. They consist of overlaps between the azadiene $\mathrm{C}=\mathrm{C}$ and $\mathrm{C}=\mathrm{N}$ double bonds and parts of the aryl rings. For clarity, these overlaps are shown separately in Figs. 6 and 7. The mean interatomic separation between the chains built around $\left(\frac{1}{2}, \frac{1}{2}, \frac{1}{2}\right)$ (Fig. 6 and Table 3) is 3.523 (5) $\AA$, while a slightly shorter separation of $3.464(5) \AA$ is observed for the second couple built around $\left(0, \frac{1}{2}, \frac{1}{2}\right)$ (Fig. 7 and Table 3$)$.

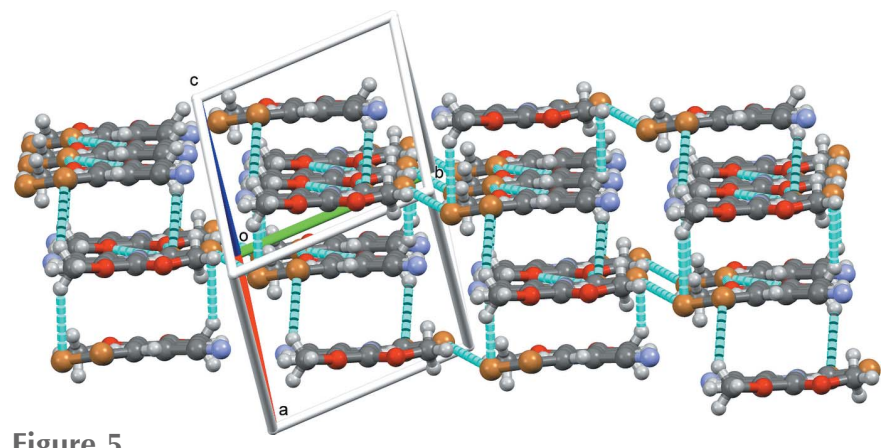

Figure 5

Part of the three-dimensional packing in (1) projected down the [101] direction and showing the halogen and weak $\mathrm{C}-\mathrm{H} \cdots \mathrm{Br}$ interactions detailed in Figs. 3 and 4.

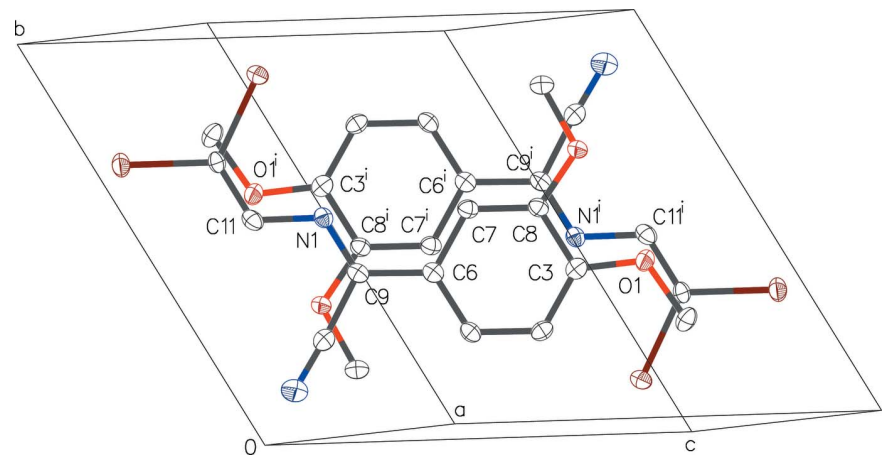

Figure 6

Part of the crystal structure of (1), showing the potential $\pi-\pi$ interactions in two head-to-tail molecules overlapping around the symmetry centre at $\left(\frac{1}{2}, \frac{1}{2}, \frac{1}{2}\right)$ (see also Fig. 4 ). H atoms have been omitted for clarity. [Symmetry code: (i) $-x+1,-y+1,-z+1$.]

\section{Database survey}

There are several other examples of structurally characterized 2-azabutadienes bearing cyano (nitrile) substituents attached at the azabutadienic array. These include 3-cyano-4-( $n$-methoxyphenyl)-1,1-diphenyl-2-aza-1,3-butadienes ( $n=2,3$ or 4$)$, 3-cyano-4-(4-cyanophenyl)-1,1-diphenyl-2-aza-1,3-butadiene, 3-cyano-4-(2,4-dimethoxyphenyl)-1,1-diphenyl-2-aza-1,3-butadiene, 3-cyano-4-(2,4-dichlorophenyl)-1,1-diphenyl-2-aza-1,3butadiene and 3-cyano-4-( $n$-fluorophenyl)-1,1-diphenyl-2-aza1,3-butadienes ( $n=2$ or 4) (Angelova et al., 1993a,b; Macícek et al., 1993a,b; Dryanska et al., 1995). Furthermore, the structure of (E)-4,4-dicyano-3-methylthio-1-phenyl-1-(1-pyrrolidinyl)-2-azabuta-1,3-diene has been reported (Lorente et al., 1996). Note that in all these structures there is a significant deviation from linearity of the $\mathrm{C}=\mathrm{N}-\mathrm{C}=\mathrm{C}$ chain. This feature is due to the presence of a substituent at the 3-C position of the 2-azabuta-1,3-diene chain. We also observed

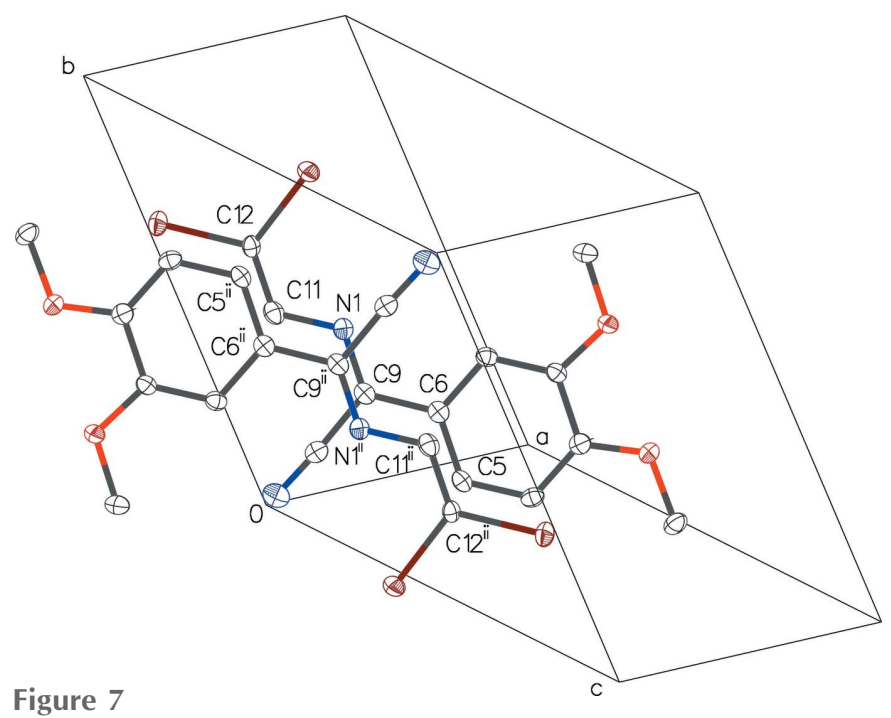

Part of the crystal structure of (1), showing the potential $\pi-\pi$ interactions in two head-to-tail molecules overlapping around the symmetry centre at $\left(0, \frac{1}{2}, \frac{1}{2}\right)$ (see also Fig. 4). H atoms have been omitted for clarity. [Symmetry code: (ii) $-x,-y+1,-z+1$.] 
and discussed this feature in the structures of $\left[\mathrm{Ar}_{2} \mathrm{C}=\mathrm{N}-\right.$ $\left.\mathrm{C}\left(\mathrm{S}^{\mathrm{t}} \mathrm{Bu}\right)=\mathrm{C}(\mathrm{H}) \mathrm{S}^{\mathrm{t}} \mathrm{Bu}\right]$ (Kinghat et al., 2016).

\section{Synthesis and crystallization}

The required $\alpha$-aminonitrile used a starting material was obtained according a literature protocol (Mai \& Patil, 1984). An equimolar mixture of $\mathrm{N}$-(dibromoethylenyl)-1-imino-1vertracetonitrile $(10 \mathrm{mmol})$ and tribromoacetaldehyde in $10 \mathrm{ml}$ of acetonitrile was stirred under reflux for $2 \mathrm{~h}$. The solution was then filtered and all volatiles removed under reduced pressure. The crude residue was recrystallized from acetonitrile affording clear-light orange crystals (yield $79 \%$; m.p. $440 \mathrm{~K} ;{ }^{1} \mathrm{H} \mathrm{RMN}\left(\mathrm{CDCl}_{3}, 300 \mathrm{MHz}\right): \delta 3.95\left(s, 3 \mathrm{H}, \mathrm{OCH}_{3}\right)$, $3.96\left(s, 3 \mathrm{H}, \mathrm{OCH}_{3}\right), 6.93(d, 1 \mathrm{H}, J=9 \mathrm{~Hz}, 1 \mathrm{Ar}-\mathrm{H}), 7.65(s, 2 \mathrm{H}$, $2 \mathrm{Ar}-\mathrm{H}), 8.04(s, 1 \mathrm{H},=\mathrm{CH}) ;{ }^{13} \mathrm{C}\left\{{ }^{1} \mathrm{H}\right\} \mathrm{NMR}\left(\mathrm{CDCl}_{3}, 75 \mathrm{MHz}\right)$ : $\delta 55.9\left(\mathrm{OCH}_{3}\right), 56.2\left(\mathrm{OCH}_{3}\right), 103.2\left(=\mathrm{CBr}_{2}\right), 110.6(C \equiv \mathrm{N})$, 124.3-153.9 $\left(C_{\mathrm{Ar}}\right), 137.8(C=\mathrm{N}), 142.2(C \mathrm{H}) ; \lambda_{\max }=245 \mathrm{~nm}$ $\left(\mathrm{e}=3300 \mathrm{M}^{-1} \mathrm{~cm}^{-1}\right), \lambda_{\max }=353 \mathrm{~nm}\left(\mathrm{e}=7580 \mathrm{M}^{-1} \mathrm{~cm}^{-1}\right)$; IR $(\mathrm{ATR}) \mathrm{cm}^{-1}: 2219(\mathrm{C} \equiv \mathrm{N}), 1597(\mathrm{C}=\mathrm{N}), 1569(\mathrm{C}=\mathrm{C})$.

\section{Refinement details}

Crystal data, data collection and structure refinement details are summarized in Table 4. All $\mathrm{H}$ atoms were placed in calculated positions and treated in a riding model. $\mathrm{C}-\mathrm{H}$ distances were set at 0.95 (aromatic) and $0.98 \AA$ (methyl), with $U_{\text {iso }}(\mathrm{H})=x U_{\text {eq }}(\mathrm{C})$, where $x=1.5$ for idealized methyl $\mathrm{H}$ atoms refined as rotating groups and 1.2 for all other $\mathrm{H}$ atoms.

\section{Acknowledgements}

We are grateful to the Universities of Franche-Comté and Bourgogne and the CNRS for financial support.

\section{References}

Angelova, O., Macíček, J. \& Dryanska, V. (1993a). Acta Cryst. C49, 1813-1818.

Angelova, O., Macíček, J. \& Dryanska, V. (1993b). Acta Cryst. C49, 1821-1823.

Bruker (2013). SAINT. Bruker AXS Inc., Madison, Wisconsin, USA.

Bruker (2014). APEX2 and SADABS. Bruker AXS Inc., Madison, Wisconsin, USA.

Cavallo, G., Metrangolo, P., Milani, R., Pilati, T., Priimagi, A., Resnati, G. \& Terraneo, G. (2016). Chem. Rev. 116, 2478-2601.

Clément, S., Guyard, L., Knorr, M., Eckert, P. K. \& Strohmann, C. (2011). Acta Cryst. E67, o481.

Dolomanov, O. V., Bourhis, L. J., Gildea, R. J., Howard, J. A. K. \& Puschmann, H. (2009). J. Appl. Cryst. 42, 339-341.

Dryanska, V., Angelova, O., Macicek, J., Shishkova, L., Denkova, P. \& Spassov, S. (1995). J. Chem. Res. pp. 268-269.

Jacquot, S., Belaissaoui, A., Schmitt, G., Laude, B., Kubicki, M. M. \& Blacque, O. (1999). Eur. J. Org. Chem. pp. 1541-1544.
Table 4

Experimental details.

\begin{tabular}{|c|c|}
\hline \multicolumn{2}{|l|}{ Crystal data } \\
\hline Chemical formula & $\mathrm{C}_{12} \mathrm{H}_{10} \mathrm{Br}_{2} \mathrm{~N}_{2} \mathrm{O}_{2}$ \\
\hline$M_{\mathrm{r}}$ & 374.04 \\
\hline Crystal system, space group & Triclinic, $P \overline{1}$ \\
\hline Temperature (K) & 100 \\
\hline$a, b, c(\AA)$ & $7.6878(4), 9.2782(5), 10.8111(6)$ \\
\hline$\alpha, \beta, \gamma\left({ }^{\circ}\right)$ & $\begin{array}{l}106.162(2), 100.887(2) \\
110.009(2)\end{array}$ \\
\hline$V\left(\AA^{3}\right)$ & $660.57(6)$ \\
\hline$Z$ & 2 \\
\hline Radiation type & Мо $K \alpha$ \\
\hline$\mu\left(\mathrm{mm}^{-1}\right)$ & 6.13 \\
\hline Crystal size $(\mathrm{mm})$ & $0.25 \times 0.2 \times 0.1$ \\
\hline \multicolumn{2}{|l|}{ Data collection } \\
\hline Diffractometer & Bruker D8 VENTURE \\
\hline Absorption correction & $\begin{array}{l}\text { Multi-scan (SADABS; Bruker, } \\
\text { 2014) }\end{array}$ \\
\hline$T_{\min }, T_{\max }$ & $0.537,0.746$ \\
\hline $\begin{array}{l}\text { No. of measured, independent and } \\
\text { observed }[I>2 \sigma(I)] \text { reflections }\end{array}$ & $23955,3045,2442$ \\
\hline$R_{\mathrm{int}}$ & 0.067 \\
\hline$(\sin \theta / \lambda)_{\max }\left(\AA^{-1}\right)$ & 0.652 \\
\hline \multicolumn{2}{|l|}{ Refinement } \\
\hline$R\left[F^{2}>2 \sigma\left(F^{2}\right)\right], w R\left(F^{2}\right), S$ & $0.027,0.067,1.03$ \\
\hline No. of reflections & 3045 \\
\hline No. of parameters & 165 \\
\hline $\mathrm{H}$-atom treatment & H-atom parameters constrained \\
\hline$\Delta \rho_{\max }, \Delta \rho_{\min }\left(\mathrm{e} \AA^{-3}\right)$ & $0.80,-0.42$ \\
\hline
\end{tabular}

Computer programs: APEX2 and SAINT (Bruker, 2013), SHELXT (Sheldrick, 2015a), SHELXL2014 (Sheldrick, 2015b) and OLEX2 (Dolomanov et al., 2009).

Jacquot, S., Schmitt, G., Laude, B., Kubicki, M. M. \& Blacque, O. (2000). Eur. J. Org. Chem. pp. 1235-1239.

Jacquot-Rousseau, S., Schmitt, G., Khatyr, A., Knorr, M., Kubicki, M. M., Vigier, E. \& Blacque, O. (2006). Eur. J. Org. Chem. pp. 2748-2751.

Kinghat, R., Boudiba, H., Khatyr, A., Knorr, M. \& Kubicki, M. M. (2008). Acta Cryst. E64, o370.

Kinghat, R., Schmitt, G., Ciamala, K., Khatyr, A., Knorr, M., JacquotRousseau, S., Rousselin, Y. \& Kubicki, M. M. (2016). C. R. Chim. 19, 319-331.

Knorr, M., Schmitt, G., Kubicki, M. M. \& Vigier, E. (2003). Eur. J. Inorg. Chem. pp. 514-517.

Lokaj, J., Moncol, J., Bures, F. \& Kulhanek, J. (2011). J. Chem. Crystallogr. 41, 834-837.

Lorente, A., Casillas, M., Gomez-Sal, P. \& Manzanero, A. (1996). Can. J. Chem. 74, 287-294.

Macíček, J., Angelova, O. \& Dryanska, V. (1993a). Acta Cryst. C49, $1818-1821$.

Macíček, J., Angelova, O. \& Dryanska, V. (1993b). Acta Cryst. C49, 2169-2173.

Mai, K. \& Patil, G. (1984). Tetrahedron Lett. 25, 4583-4586.

Sato, N. \& Adachi, J. (1978). J. Org. Chem. 43, 340-341.

Sheldrick, G. M. (2015a). Acta Cryst. A71, 3-8.

Sheldrick, G. M. (2015b). Acta Cryst. C71, 3-8. 


\section{supporting information}

Acta Cryst. (2016). E72, 1167-1170 [https://doi.org/10.1107/S2056989016011075]

Crystal structure of 4,4-dibromo-1-(3,4-dimethoxyphenyl)-2-azabuta-1,3diene-1-carbonitrile

Marwa Chaabene, Abderrahim Khatyr, Michael Knorr, Moheddine Askri, Yoann Rousselin and

Marek M. Kubicki

Computing details

Data collection: APEX2 (Bruker, 2014); cell refinement: SAINT (Bruker, 2013); data reduction: SAINT (Bruker, 2013); program(s) used to solve structure: SHELXT (Sheldrick, 2015a); program(s) used to refine structure: SHELXL2014 (Sheldrick, 2015b); molecular graphics: OLEX2 (Dolomanov et al., 2009); software used to prepare material for publication: OLEX2 (Dolomanov et al., 2009).

4,4-Dibromo-1-(3,4-dimethoxyphenyl)-2-azabuta-1,3-diene-1-carbonitrile

Crystal data

$\mathrm{C}_{12} \mathrm{H}_{10} \mathrm{Br}_{2} \mathrm{~N}_{2} \mathrm{O}_{2}$

$M_{r}=374.04$

Triclinic, $P \overline{1}$

$a=7.6878(4) \AA$

$b=9.2782(5) \AA$

$c=10.8111(6) \AA$

$\alpha=106.162(2)^{\circ}$

$\beta=100.887(2)^{\circ}$

$\gamma=110.009(2)^{\circ}$

$V=660.57(6) \AA^{3}$

\section{Data collection}

Bruker D8 VENTURE diffractometer

Radiation source: X-ray tube, Siemens KFF Mo 2K-90C

TRIUMPH curved crystal monochromator $\varphi$ and $\omega$ scans'

Absorption correction: multi-scan

(SADABS; Bruker, 2014)

$T_{\min }=0.537, T_{\max }=0.746$

Refinement

Refinement on $F^{2}$

Least-squares matrix: full

$R\left[F^{2}>2 \sigma\left(F^{2}\right)\right]=0.027$

$w R\left(F^{2}\right)=0.067$

$S=1.03$

3045 reflections
$Z=2$

$F(000)=364$

$D_{\mathrm{x}}=1.881 \mathrm{Mg} \mathrm{m}^{-3}$

Mo $K \alpha$ radiation, $\lambda=0.71073 \AA$

Cell parameters from 8732 reflections

$\theta=3.0-27.5^{\circ}$

$\mu=6.13 \mathrm{~mm}^{-1}$

$T=100 \mathrm{~K}$

Plqte, clear light orange

$0.25 \times 0.2 \times 0.1 \mathrm{~mm}$

23955 measured reflections

3045 independent reflections

2442 reflections with $I>2 \sigma(I)$

$R_{\text {int }}=0.067$

$\theta_{\text {max }}=27.6^{\circ}, \theta_{\min }=3.0^{\circ}$

$h=-9 \rightarrow 10$

$k=-12 \rightarrow 12$

$l=-14 \rightarrow 14$

165 parameters

0 restraints

Primary atom site location: structure-invariant direct methods

Hydrogen site location: inferred from neighbouring sites 
H-atom parameters constrained

$w=1 /\left[\sigma^{2}\left(F_{\mathrm{o}}^{2}\right)+(0.0307 P)^{2}+0.5386 P\right]$

where $P=\left(F_{\mathrm{o}}^{2}+2 F_{\mathrm{c}}{ }^{2}\right) / 3$

$$
\begin{aligned}
& (\Delta / \sigma)_{\max }=0.001 \\
& \Delta \rho_{\max }=0.80 \mathrm{e} \AA^{-3} \\
& \Delta \rho_{\min }=-0.42 \mathrm{e} \AA^{-3}
\end{aligned}
$$

Special details

Experimental. Absorption correction: SADABS-2014/4 (Bruker,2014) was used for absorption correction. wR2(int) was 0.0938 before and 0.0647 after correction. The Ratio of minimum to maximum transmission is 0.7197 . The $\lambda / 2$ correction factor is 0.00150 .

Geometry. All esds (except the esd in the dihedral angle between two 1.s. planes) are estimated using the full covariance matrix. The cell esds are taken into account individually in the estimation of esds in distances, angles and torsion angles; correlations between esds in cell parameters are only used when they are defined by crystal symmetry. An approximate

\begin{tabular}{|c|c|c|c|c|}
\hline & $x$ & $y$ & $z$ & $U_{\text {iso }} * / U_{\text {eq }}$ \\
\hline $\mathrm{C} 1$ & $0.5229(4)$ & $0.2556(4)$ & $0.9031(3)$ & $0.0198(6)$ \\
\hline H1A & 0.5402 & 0.1716 & 0.8346 & $0.030^{*}$ \\
\hline H1B & 0.3876 & 0.2129 & 0.9037 & $0.030 *$ \\
\hline $\mathrm{H} 1 \mathrm{C}$ & 0.6109 & 0.2824 & 0.9927 & $0.030^{*}$ \\
\hline $\mathrm{C} 2$ & $0.6815(5)$ & $0.8341(4)$ & 0.8299 & $0.0246(7)$ \\
\hline $\mathrm{H} 2 \mathrm{~A}$ & 0.7114 & 0.8389 & 0.7465 & $0.037^{*}$ \\
\hline $\mathrm{H} 2 \mathrm{~B}$ & 0.7909 & 0.9196 & 0.9088 & $0.037^{*}$ \\
\hline $\mathrm{H} 2 \mathrm{C}$ & 0.5636 & 0.8527 & 0.8307 & $0.037^{*}$ \\
\hline $\mathrm{C} 3$ & $0.4518(4)$ & 0.3908 & $0.7558(3)$ & $0.0163(6)$ \\
\hline $\mathrm{C} 4$ & $0.3008(4)$ & 0.2473 & $0.6590(3)$ & $0.0160(6)$ \\
\hline $\mathrm{H} 4$ & 0.2729 & 0.1450 & 0.6709 & $0.019 *$ \\
\hline $\mathrm{C} 5$ & 0.1904 (4) & 0.2535 & $0.5444(3)$ & $0.0156(6)$ \\
\hline H5 & 0.0857 & 0.1552 & 0.4792 & $0.019^{*}$ \\
\hline C6 & $0.2308(4)$ & $0.4007(3)$ & $0.5242(3)$ & $0.0149(5)$ \\
\hline $\mathrm{C} 7$ & $0.3852(4)$ & $0.5476(3)$ & $0.6221(3)$ & $0.0154(6)$ \\
\hline H7 & 0.4130 & 0.6496 & 0.6096 & $0.019^{*}$ \\
\hline $\mathrm{C} 8$ & $0.4946(4)$ & $0.5419(3)$ & $0.7352(3)$ & $0.0149(5)$ \\
\hline C9 & $0.1186(4)$ & $0.4092(3)$ & 0.4029 & $0.0148(5)$ \\
\hline $\mathrm{C} 10$ & $-0.0396(4)$ & $0.2543(4)$ & 0.3038 & $0.0210(6)$ \\
\hline $\mathrm{C} 11$ & 0.0555 (4) & $0.5495(3)$ & $0.2655(3)$ & $0.0175(6)$ \\
\hline H11 & -0.0400 & 0.4495 & 0.1958 & $0.021 *$ \\
\hline $\mathrm{C} 12$ & $0.0911(4)$ & $0.6927(3)$ & 0.2490 & $0.0139(5)$ \\
\hline N1 & $0.1576(3)$ & 0.5463 & $0.3839(2)$ & $0.0145(5)$ \\
\hline N2 & $-0.1661(4)$ & $0.1374(3)$ & 0.2229 & $0.0356(7)$ \\
\hline $\mathrm{O} 1$ & $0.5666(3)$ & $0.4013(2)$ & $0.8715(2)$ & 0.0195 (4) \\
\hline $\mathrm{O} 2$ & $0.6500(3)$ & $0.6752(2)$ & $0.8351(2)$ & $0.0195(4)$ \\
\hline Br1 & $0.27146(4)$ & $0.89631(3)$ & 0.38235 (3) & $0.01976(9)$ \\
\hline $\mathrm{Br} 2$ & $-0.04365(4)$ & 0.69810 & $0.08743(3)$ & $0.01900(9)$ \\
\hline
\end{tabular}
(isotropic) treatment of cell esds is used for estimating esds involving 1.s. planes.

Fractional atomic coordinates and isotropic or equivalent isotropic displacement parameters $\left(\AA^{2}\right)$

Atomic displacement parameters $\left(\AA^{2}\right)$

\begin{tabular}{lllllll}
\hline & $U^{11}$ & $U^{22}$ & $U^{33}$ & $U^{12}$ & $U^{13}$ & $U^{23}$ \\
\hline $\mathrm{C} 1$ & $0.0209(14)$ & $0.0221(14)$ & $0.0233(16)$ & $0.0103(12)$ & $0.0097(12)$ & $0.0148(13)$
\end{tabular}




$\begin{array}{lllllll}\text { C2 } & 0.0258(16) & 0.0142(14) & 0.0254(17) & 0.0023(12) & 0.0012(13) & 0.0074(13) \\ \text { C3 } & 0.0145(13) & 0.0177(14) & 0.0202(15) & 0.0082(11) & 0.0082(11) & 0.0086(12) \\ \text { C4 } & 0.0180(14) & 0.0152(13) & 0.0194(15) & 0.0087(11) & 0.0095(12) & 0.0083(11) \\ \text { C5 } & 0.0121(13) & 0.0167(13) & 0.0147(14) & 0.0043(11) & 0.0038(11) & 0.0035(11) \\ \text { C6 } & 0.0142(12) & 0.0161(13) & 0.0146(14) & 0.0071(10) & 0.0062(11) & 0.0038(11) \\ \text { C7 } & 0.0168(13) & 0.0137(13) & 0.0144(14) & 0.0050(11) & 0.0058(11) & 0.0044(11) \\ \text { C8 } & 0.0132(13) & 0.0142(13) & 0.0152(14) & 0.0039(10) & 0.0046(11) & 0.0048(11) \\ \text { C9 } & 0.0133(13) & 0.0151(13) & 0.0146(14) & 0.0061(10) & 0.0055(11) & 0.0025(11) \\ \text { C10 } & 0.0226(15) & 0.0186(14) & 0.0217(16) & 0.0076(13) & 0.0050(13) & 0.0099(13) \\ \text { C11 } & 0.0171(14) & 0.0170(14) & 0.0141(14) & 0.0069(11) & 0.0033(11) & 0.0012(11) \\ \text { C12 } & 0.0133(12) & 0.0179(13) & 0.0104(13) & 0.0064(11) & 0.0042(11) & 0.0053(11) \\ \text { N1 } & 0.0157(11) & 0.0160(11) & 0.0117(12) & 0.0072(9) & 0.0049(9) & 0.0038(9) \\ \text { N2 } & 0.0337(16) & 0.0205(14) & 0.0371(18) & 0.0036(12) & -0.0042(14) & 0.0087(13) \\ \text { O1 } & 0.0191(10) & 0.0185(10) & 0.0202(11) & 0.0059(8) & 0.0025(8) & 0.0110(9) \\ \text { O2 } & 0.0203(10) & 0.0130(9) & 0.0174(11) & 0.0017(8) & -0.0006(8) & 0.0053(8) \\ \text { Br1 } & 0.02102(15) & 0.01428(14) & 0.01661(16) & 0.00322(11) & 0.00125(12) & 0.00357(11) \\ \text { Br2 } & 0.02026(15) & 0.02270(16) & 0.01424(16) & 0.01035(12) & 0.00265(12) & 0.00730(12)\end{array}$

Geometric parameters $\left(\AA,{ }^{\circ}\right)$

\begin{tabular}{llll}
\hline $\mathrm{C} 1-\mathrm{H} 1 \mathrm{~A}$ & 0.9800 & $\mathrm{C} 5-\mathrm{C} 6$ & $1.383(4)$ \\
$\mathrm{C} 1-\mathrm{H} 1 \mathrm{~B}$ & 0.9800 & $\mathrm{C} 6-\mathrm{C} 7$ & $1.415(4)$ \\
$\mathrm{C} 1-\mathrm{H} 1 \mathrm{C}$ & 0.9800 & $\mathrm{C} 6-\mathrm{C} 9$ & $1.464(4)$ \\
$\mathrm{C} 1-\mathrm{O} 1$ & $1.431(3)$ & $\mathrm{C} 7-\mathrm{H} 7$ & 0.9500 \\
$\mathrm{C} 2-\mathrm{H} 2 \mathrm{~A}$ & 0.9800 & $\mathrm{C} 7-\mathrm{C} 8$ & $1.372(4)$ \\
$\mathrm{C} 2-\mathrm{H} 2 \mathrm{~B}$ & 0.9800 & $\mathrm{C} 8-\mathrm{O} 2$ & $1.372(3)$ \\
$\mathrm{C} 2-\mathrm{H} 2 \mathrm{C}$ & 0.9800 & $\mathrm{C} 9-\mathrm{C} 10$ & $1.465(4)$ \\
$\mathrm{C} 2-\mathrm{O} 2$ & $1.430(3)$ & $\mathrm{C} 9-\mathrm{N} 1$ & $1.288(3)$ \\
$\mathrm{C} 3-\mathrm{C} 4$ & $1.387(4)$ & $\mathrm{C} 10-\mathrm{N} 2$ & $1.147(4)$ \\
$\mathrm{C} 3-\mathrm{C} 8$ & $1.418(4)$ & $\mathrm{C} 11-\mathrm{H} 11$ & 0.9500 \\
$\mathrm{C} 3-\mathrm{O} 1$ & $1.345(3)$ & $\mathrm{C} 11-\mathrm{C} 12$ & $1.332(4)$ \\
$\mathrm{C} 4-\mathrm{H} 4$ & 0.9500 & $\mathrm{C} 11-\mathrm{N} 1$ & $1.384(4)$ \\
$\mathrm{C} 4-\mathrm{C} 5$ & $1.391(4)$ & $\mathrm{C} 12-\mathrm{B} 1$ & $1.872(3)$ \\
$\mathrm{C} 5-\mathrm{H} 5$ & 0.9500 & $\mathrm{C} 12-\mathrm{B} 2$ & $1.878(3)$ \\
& & & $121.7(2)$ \\
$\mathrm{H} 1 \mathrm{~A}-\mathrm{C} 1-\mathrm{H} 1 \mathrm{~B}$ & 109.5 & $\mathrm{C} 5-\mathrm{C} 6-\mathrm{C} 9$ & $118.7(2)$ \\
$\mathrm{H} 1 \mathrm{~A}-\mathrm{C} 1-\mathrm{H} 1 \mathrm{C}$ & 109.5 & $\mathrm{C} 7-\mathrm{C} 6-\mathrm{C} 9$ & 120.2 \\
$\mathrm{H} 1 \mathrm{~B}-\mathrm{C} 1-\mathrm{H} 1 \mathrm{C}$ & 109.5 & $\mathrm{C} 6-\mathrm{C} 7-\mathrm{H} 7$ & $119.6(3)$ \\
$\mathrm{O} 1-\mathrm{C} 1-\mathrm{H} 1 \mathrm{~A}$ & 109.5 & $\mathrm{C} 8-\mathrm{C} 7-\mathrm{C} 6$ & 120.2 \\
$\mathrm{O} 1-\mathrm{C} 1-\mathrm{H} 1 \mathrm{~B}$ & 109.5 & $\mathrm{C} 8-\mathrm{C} 7-\mathrm{H} 7$ & $120.5(3)$ \\
$\mathrm{O} 1-\mathrm{C} 1-\mathrm{H} 1 \mathrm{C}$ & 109.5 & $\mathrm{C} 7-\mathrm{C} 8-\mathrm{C} 3$ & $114.7(2)$ \\
$\mathrm{H} 2 \mathrm{~A}-\mathrm{C} 2-\mathrm{H} 2 \mathrm{~B}$ & 109.5 & $\mathrm{O} 2-\mathrm{C} 8-\mathrm{C} 3-\mathrm{C} 7$ & $124.8(2)$ \\
$\mathrm{H} 2 \mathrm{~A}-\mathrm{C} 2-\mathrm{H} 2 \mathrm{C}$ & 109.5 & $\mathrm{C} 6-\mathrm{C} 9-\mathrm{C} 10$ & $117.0(2)$ \\
$\mathrm{H} 2 \mathrm{~B}-\mathrm{C} 2-\mathrm{H} 2 \mathrm{C}$ & 109.5 & $\mathrm{~N} 1-\mathrm{C} 9-\mathrm{C} 6$ & $121.6(2)$ \\
$\mathrm{O} 2-\mathrm{C} 2-\mathrm{H} 2 \mathrm{~A}$ & 109.5 & $\mathrm{~N} 1-\mathrm{C} 9-\mathrm{C} 10$ & $121.5(3)$ \\
$\mathrm{O} 2-\mathrm{C} 2-\mathrm{C} 10-\mathrm{C} 9$ & $176.7(3)$ \\
$\mathrm{O} 2-\mathrm{C} 2-\mathrm{H} 2 \mathrm{C}$ & 109.5 & $\mathrm{C} 12-\mathrm{C} 11-\mathrm{H} 11$ & 120.0 \\
$\mathrm{C} 4-\mathrm{C} 3-\mathrm{C} 8$ & 109.5 & &
\end{tabular}




$\begin{array}{llll}\mathrm{O} 1-\mathrm{C} 3-\mathrm{C} 4 & 125.4(3) & \mathrm{C} 12-\mathrm{C} 11-\mathrm{N} 1 & 120.0(3) \\ \mathrm{O} 1-\mathrm{C} 3-\mathrm{C} 8 & 115.1(2) & \mathrm{N} 1-\mathrm{C} 11-\mathrm{H} 11 & 120.0 \\ \mathrm{C} 3-\mathrm{C} 4-\mathrm{H} 4 & 120.1 & \mathrm{C} 11-\mathrm{C} 12-\mathrm{Br} 1 & 123.0(2) \\ \mathrm{C} 3-\mathrm{C} 4-\mathrm{C} 5 & 119.8(3) & \mathrm{C} 11-\mathrm{C} 12-\mathrm{Br} 2 & 120.4(2) \\ \mathrm{C} 5-\mathrm{C} 4-\mathrm{H} 4 & 120.1 & \mathrm{~B} 1-\mathrm{C} 12-\mathrm{Br} 2 & 116.65(14) \\ \mathrm{C} 4-\mathrm{C} 5-\mathrm{H} 5 & 119.5 & \mathrm{C} 9-\mathrm{N} 1-\mathrm{C} 11 & 120.4(2) \\ \mathrm{C} 6-\mathrm{C} 5-\mathrm{C} 4 & 120.9(3) & \mathrm{C} 3-\mathrm{O} 1-\mathrm{C} 1 & 118.0(2) \\ \mathrm{C} 6-\mathrm{C} 5-\mathrm{H} 5 & 119.5 & \mathrm{C} 8-\mathrm{O} 2-\mathrm{C} 2 & 116.8(2) \\ \mathrm{C} 5-\mathrm{C} 6-\mathrm{C} 7 & 119.6(3) & & \\ & & \mathrm{C} 7-\mathrm{C} 6-\mathrm{C} 9-\mathrm{C} 10 & 179.8(2) \\ \mathrm{C} 3-\mathrm{C} 4-\mathrm{C} 5-\mathrm{C} 6 & 1.1(4) & \mathrm{C} 7-\mathrm{C} 6-\mathrm{C} 9-\mathrm{N} 1 & 0.0(4) \\ \mathrm{C} 3-\mathrm{C} 8-\mathrm{O} 2-\mathrm{C} 2 & -171.0(2) & \mathrm{C} 7-\mathrm{C} 8-\mathrm{O} 2-\mathrm{C} 2 & -1.4(4) \\ \mathrm{C} 4-\mathrm{C} 3-\mathrm{C} 8-\mathrm{C} 7 & 1.5(4) & \mathrm{C} 8-\mathrm{C} 3-\mathrm{C} 4-\mathrm{C} 5 & 175.8(2) \\ \mathrm{C} 4-\mathrm{C} 3-\mathrm{C} 8-\mathrm{O} 2 & -178.2(2) & \mathrm{C} 9-\mathrm{C} 3-\mathrm{O} 1-\mathrm{C} 7-\mathrm{C} 8 & -178.7(2) \\ \mathrm{C} 4-\mathrm{C} 3-\mathrm{O} 1-\mathrm{C} 1 & -4.4(4) & \mathrm{C} 10-\mathrm{C} 9-\mathrm{N} 1-\mathrm{C} 11 & -2.9(4) \\ \mathrm{C} 4-\mathrm{C} 5-\mathrm{C} 6-\mathrm{C} 7 & -0.7(4) & \mathrm{C} 12-\mathrm{C} 11-\mathrm{N} 1-\mathrm{C} 9 & 177.2(3) \\ \mathrm{C} 4-\mathrm{C} 5-\mathrm{C} 6-\mathrm{C} 9 & 178.7(3) & \mathrm{N} 1-\mathrm{C} 11-\mathrm{C} 12-\mathrm{Br} 1 & -1.7(4) \\ \mathrm{C} 5-\mathrm{C} 6-\mathrm{C} 7-\mathrm{C} 8 & 0.8(4) & \mathrm{N} 1-\mathrm{C} 11-\mathrm{C} 12-\mathrm{Br} 2 & 179.0(2) \\ \mathrm{C} 5-\mathrm{C} 6-\mathrm{C} 9-\mathrm{C} 10 & 0.3(4) & \mathrm{O} 1-\mathrm{C} 3-\mathrm{C} 4-\mathrm{C} 5 & 178.8(3) \\ \mathrm{C} 5-\mathrm{C} 6-\mathrm{C} 9-\mathrm{N} 1 & -179.5(3) & \mathrm{O} 1-\mathrm{C} 3-\mathrm{C} 8-\mathrm{C} 7 & -178.7(3) \\ \mathrm{C} 6-\mathrm{C} 7-\mathrm{C} 8-\mathrm{C} 3 & -1.2(4) & \mathrm{O} 1-\mathrm{C} 3-\mathrm{C} 8-\mathrm{O} 2 & 1.5(4) \\ \mathrm{C} 6-\mathrm{C} 7-\mathrm{C} 8-\mathrm{O} 2 & 178.5(2) & 176.9(2) & \end{array}$

Hydrogen-bond geometry $\left(A,{ }^{\circ}\right)$

\begin{tabular}{lllll}
\hline$D-\mathrm{H} \cdots A$ & $D-\mathrm{H}$ & $\mathrm{H} \cdots A$ & $D \cdots A$ & $D-\mathrm{H} \cdots A$ \\
\hline $\mathrm{C} 1-\mathrm{H} 1 A \cdots \mathrm{Br} 1^{\mathrm{i}}$ & 0.98 & 3.01 & $3.867(4)$ & 146 \\
$\mathrm{C} 1-\mathrm{H} 1 B \cdots \mathrm{Br} 2^{\mathrm{ii}}$ & 0.98 & 3.04 & $3.869(4)$ & 143 \\
\hline
\end{tabular}

Symmetry codes: (i) $-x+1,-y+1,-z+1$; (ii) $-x,-y+1,-z+1$. 